\title{
Wien oder das letzte Fest der Aufklärung
}

\section{Engelhard Weigl}

"Wien sollte in Deutschland sein, was Paris in Frankreich ist, und wir alle sollten in Wien sein. Das wäre eine herrliche Sache." Wieland an Riedel 1765

\section{Reisende nach Wien}

Die Menge der Fuhrwerke und Frachtwagen, die auf den Straßen verkehrten, zeigten dem erfahrenen Reisenden an, daß er sich einer großen Stadt näherte. In Hollabrunn - wenige Meilen vor Wien - legte Georg Forster noch eine Übernachtung ein, um am nächsten Tag rechtzeitig in der Hauptstadt der Habsburger Monarchie anzukommen. "Schöner mondheller, linder Abend," notiert er in seinem Tagebuch "wobei ich über die Entstehung der Städte und ihr Verhältnis zur Aufklärung nachdachte."1 Über das Verhältnis der Städte zur Aufklärung enthält das Tagebuch keine weiteren Bemerkungen, doch hat Forster während der sieben ereignisreichen Wochen, die er sich in Wien aufgehalten hat, sorgfältig Tagebuch geführt, sodaß wir seinen Aufenthalt als einen Einstieg für unsere zentrale Frage nach dem Verhältnis von Aufklärung und Stadtkultur benutzen können. Die Stadt Wien wird sich also für uns aus der Perspektive eines Reisenden erschließen, der, aus Göttingen kommend, verblüfft und begeistert in den Wirbel eines gesellschaftlichen Lebens eintaucht, das auf gänzlich anderen kulturellen Voraussetzungen beruht als der protestantische Norden Deutschlands.

Für Forster, der durch seine Teilnahme an Cooks zweiter Weltumsegelung (1772-75) und durch seinen Reisebericht Voyage round the World (1776) zu einer europäischen Berühmtheit geworden war, wurde sein Wienaufenthalt zu einem Höhepunkt seines Lebens. "Wien, liebster Bruder," schreibt er seinem Freund Samuel Thomas Sömmerling, "ist der Ort, der unter allen mich noch am meisten gefesselt hat."2 Dieser Satz

${ }^{1}$ Georg Forsters Werke, hrsg. von der Akademie der Wissenschaften, 12. Bd., Tagebücher, Berlin 1973, S. 103.

${ }^{2}$ Georg Forsters Werke, 14. Bd., Briefe 1784-Juni 1787, Berlin 1978, S. 171. 
bekommt erst sein richtiges Gewicht, wenn man bedenkt, daß der beinahe dreißigjährige Forster bereits Paris und London gesehen hatte und von den deutschen Zentren der Aufklärung Leipzig, Halle und Göttingen, dazu die Küsten von Neuseeland und die das Europa der Aufklärung bezaubernde Südseeinsel Tahiti. Ob in Tahiti oder in Wien, Forsters Hauptaugenmerk war auf das soziale Verhalten der Menschen und Menschengruppen gerichtet. In Wien findet der Kosmopolit Forster kosmopolitische Formen des Umganges, eine die Gegensätze der Stände und Nationen, Rassen und Religionen überwindende Geselligkeit, die alles bisher erlebte weit übertrifft. "Für ein Herz, wie das meinige, welches der Freude mit Menschen $\mathrm{zu}$ leben, sie zu lieben und von ihnen geliebt zu werden, so offen ist, muß ich dir gestehen, ist Wien ein Paradies."3

Forsters Reiseeindrücke sind nicht singulär. Unter den Reisenden, die es in den ersten Regierungsjahren von Kaiser Joseph II. nach Wien zog, ist Mozart der berühmteste. In Wien wagt er den Sprung in die damals grundsätzlich neue Existenzform des freien Künstlers, der für einen freien Markt von Auftraggebern auf eigenes Risiko arbeitet. Keine Stadt in Europa bot dafür nach seinem Urteil günstigere Voraussetzungen. Kaum drei Wochen in Wien schreibt er voller Zuversicht an seinen Vater: "ich versichere sie, daß hier ein Herrlicher ort ist - und für mein Metier der beste ort von der Welt. - das wird ihnen Jederman sagen."4

Auch in Preußen rückte nach dem Regierungsantritt des neuen Kaisers Wien in den Blickpunkt des Interesses. "Maria Theresia ist nicht mehr eine neue Ordnung beginnt." Mit diesem Satz hatte Friedrich der Große 1780 eine neue Epoche für Österreich proklamiert und alle Zeichen sprachen dafür, daß sich große Veränderungen vollzogen. Von Berlin aus machte sich der Großverleger und Publizist, Freund Moses Mendelssohns und Lessings, Friedrich Nicolai am 1. Mai 1781 Richtung Wien auf die Reise, um den Entwicklungsstand von Aufklärung und Industrie im unbekannten Süden Deutschlands zu prüfen. Gerüstet mit einem neuen Wegmesser an seiner Kutsche, um die zurüickgelegten Meilen exakt zu bestimmen und einer neuerfundenen tragbaren Schreibfeder mit Tinte, einer Art Füllfederhalter, die es erlaubte, an jedem Ort der Reise Notizen

${ }^{3}$ Ebd., S. 172 .

${ }^{4}$ Mozart: Briefe und Aufzeichnungen, Gesamtausgabe, hrsg. von Wilhelm A. Bauer u. Otto Erich Deutsch, Bd. III, Kassel, Basel etc. 1963, S. 102. Mozart am 4. April an seinen Vater. 
zu machen, nimmt sich Nicolai vor, die neuen Standards der Rationalität auch auf den katholischen Süden anzuwenden, um die Deutschen besser mit Deutschland bekannt zu machen. "Die Stadt Wien, die größte in Deutschland, die schon vorher so wichtig und merkwürdig war, wird jetzt noch merkwürdiger, durch den Geist des wohltätigen Monarchen, der seine Untertanen aus dem Schlummer, in welchem sie bisher gelegen hatten, zu erwecken sucht, und Verbesserungen von aller Art veranlaßt. Die wahre Beschaffenheit Wiens ist in dem nördlichen Deutschlande nicht sehr bekannt. Ich werde also über diese Residenzstadt weitläufiger sein müssen, als über andere Städte, die ich auf meiner Reise besucht habe." 5 1500 Seiten seiner sechsbändigen Reisebeschreibung widmet Nicolai der Hauptstadt des Habsburgischen Reiches und verleiht ihr allein dadurch eine Bedeutung, die sich für den Berliner nur mit der Berlins vergleichen läßt.

Unter den Reisenden nach Wien befand sich auch der heute weitgehend vergessene Schriftsteller Johann Pezzl (1756-1822), der aus Bayern stammend, 1784 in Wien ankam. 1783 veröffentlichte Pezzl in Zürich den Roman Faustin, oder das philosophische Jahrhundert, der zum ersten durchschlagenden Erfolgsroman der österreichischen Aufklärung werden sollte und die Attraktion Wiens für die bürgerlichen Intellektuellen und ihre utopischen Hoffnungen bereits reflektiert. Unter den fünfzig Büchern, die sich in Mozarts schmaler persönlicher Bibliothek befanden, wurde nach seinem Tode auch dieser populäre Roman gefunden. Pezzl erzählt in diesem Roman die Geschichte eines katholischen Candide, der wie sein berühmtes Vorbild durch die ganze Welt reist, um die Behauptung seines Lehrers zu überprüfen, daß seit 1748 die Morgenröte der Aufklärung in Europa angebrochen sei. Doch, was Faustin erfährt, ist überall das reine Gegenteil von Vernunft, Toleranz und Menschenliebe. Sein Vater wird von Bauern erschlagen, die rebellieren, weil Feiertage im Sinne der neuen rationalen Ökonomie abgeschafft werden, sein Lehrer Pater Bonifaz wird ins Klostergefängnis gesperrt, weil er Bücher von Voltaire, Helvetius und Bayle besitzt. Faustin reist von München nach Ingolstadt und über Venedig und Rom nach Neapel, besucht Amerika und England, um überall die ungebrochene Macht des Aberglaubens und der Intoleranz zu

${ }^{5}$ Friedrich Nicolai: Beschreibung einer Reise durch Deutschland und die Schweiz, im Jahre 1781. Nebst Bemerkungen über Gelehrsamkeit, Industrie, Religion und Sitten, 1. Bd., Berlin und Stettin 1783, S. 571. 
erfahren. Doch anders als Voltaires Candide findet Faustin seinen Frieden nicht in der Abgeschiedenheit des Gartens fern vom Treiben der Menschen, sondern in der einzigen Großstadt des Reiches - in Wien. 1780, das Jahr des Regierungsantritts. Joseph II. wird von Faustin als Beginn des philosophischen Jahrhunderts, als Sieg der Aufklärung erlebt. "Unter all dem Lesen, Bewundern und Beloben waren sie endlich in Wien selbst angelangt." heißt es im letzten Kapitel des Romans: "Die Philosophie auf dem Thron": "Ihr erster Gang war nun in den Augarten. ${ }^{6}$ Faustin las die Aufschrift ober [über] dem Eingang, die Joseph beigesetzt: Allen Menschen gewidmeter Belustigungsort von ihrem Schätzer. - Heiliger Hain! rief er bei Ansicht dieser Aufschrift: Wonniges Denkmal der Philosophie auf dem Thron! wie glücklich sind wir, unter deinem Schatten, in der Nähe des erhabenen Schätzers der Menschheit wandeln zu können! Diese Aufschrift war ihm schöner als alle, die je eine Akademie der [an] Inschriften zur Welt gebracht hat." Faustins Begleiter zählt nun die lange Liste der "schönen Taten" des großen Kaisers auf, die von ihm "zur Erleuchtung, Beförderung der Toleranz und Umschaffung der NationalDenkart" unternommen wurden. Dazu zählt die weitgehende Lockerung der Zensur, die umfangreiche Reform der Kirche, "Toleranz-Edikte" für die ganze Monarchie, Verbesserung der Stadt- und Landschulen und des Justizwesens. "So flossen ihnen die ersten Tage dahin. Je länger sie da waren, je mehr Beweise von dem schöpferischen Umschwung der ehedem finstern und eingeschränkten Denkart des Wiener Publikums entdeckten sie. Faustin tat Traubach den Vorschlag, sie wollten in ihrem Briefwechsel mit ihren Freunden eine neue Ära anfangen, die Ära des aufgeklärten südlichen Deutschlands, die Josephische Ära, und das Jahr 1780 zum Stufenjahr derselben festsetzen."

Unter Joseph II. wurde Wien für eine kurze Periode von zehn fieberhaften Jahren zur freiesten, offensten, liberalsten und tolerantesten Stadt in Europa. Wien versprach der Sitz einer erneuerten deutschen Kultur zu werden, bei der das Theater und die Oper eine zentrale Rolle spielen

${ }^{6}$ Der kaiserliche Augarten - Erholungsort der Wiener, wo man an schönen Nachmittagen im Sommer spazieren ging, um zu sehen und gesehen $\mathrm{zu}$ werden - wurde 1775 von Joseph II. der Öffentlichkeit zur Verfügung gestellt.

7 Johann Pezzl: Faustin oder das philosophische Jahrhundert, Zürich 1783, S. $366 \mathrm{ff}$. 
sollten. So übertrieben uns heute die Proklamation einer neuen Epoche durch Pezzl anmutet, so stand er mit seinem Enthusiasmus nicht allein. Klopstock widmete Joseph II. 1781 die Ode An den Kaiser, ebenfalls sein nationales Drama Hermainn Schlacht. Wieland dedizierte ihm seinen Roman Der goldene Spiegel (1772), in dem er den aufgeklärten Absolutismus mit utopischen Zügen versah. Selbst Lessing, der 1778 mit Joseph II. bald aufgeklärtere und tugendhaftere Zeiten heraufkommen sah, ${ }^{8}$ versuchte in Wien Fuß zu fassen. Seine Verbitterung gegenüber dem Berlin Friedrichs des Großen verführte auch den kritischsten Geist Deutschlands zu überzogenen Hoffnungen. Noch während der Regierungszeit Maria-Theresias schreibt er - den Lokalpatriotismus seiner Berliner Freunde bewußt verletzend -: "Wien mag sein, wie es will, der deutschen Literatur verspreche ich dort immer noch mehr Glück, als in Eurem französisierten Berlin. Wenn der "Phädon" in Wien konfisziert ist: so muß es bloß geschehen sein, weil er in Berlin gedruckt worden, und man sich nicht einbilden können, daß man in Berlin für die Ünsterblichkeit der Seele schreibe. Sonst sagen Sie mir von Ihrer Berlinischen Freiheit zu denken und zu schreiben ja nichts. Sie reduziert sich einzig und alleine auf die Freiheit, gegen die Religion so viel Sottisen zu Markt zu bringen, als man will. [...] Lassen Sie es aber doch einmal einen in Berlin versuchen, über andere Dinge so frei zu schreiben, als Sonnenfels in Wien geschrieben hat; lassen Sie es ihn versuchen, dem vornehmen Hofpöbel so die Wahrheit zu sagen, als dieser sie ihm gesagt hat; lassen sie einen in Berlin auftreten, der für die Rechte der Untertanen, der gegen Aussaugung und Despotismus seine Stimme erheben wollte, [...] und sie werden bald die Erfahrung haben, welches Land bis auf den heutigen Tag das sklavischste Land von Europa ist."9 1769 und 1775 reiste Lessing nach Wien und wurde von Maria-Theresia und Joseph II. empfangen und hoffte - wenn auch vergeblich - auf eine Ernennung zum Direktor des geplanten Nationaltheaters.

${ }^{8}$ Gotthold Ephraim Lessing: "Anti-Goeze" in: G. E. Lessing: Gesammelte Werke, hrsg. von P. Rilla, Berlin und Weimar 1968, Bd. 8, S. 204.

${ }^{9}$ Lessing an Friedrich Nicolai, Hamburg, den 25. August 1769. 


\section{Die Reformpolitik Josephs II.}

Der Glanz, der für die bürgerlichen Intellektuellen von Wien ausging, war in hohem Maße von der faszinierenden Persönlichkeit des Kaisers bestimmt, der nach dem Tode seiner Mutter Maria-Theresia $1780 \mathrm{mit}$ einem Bündel von Maßnahmen hervortrat, die alle zu den Wunschvorstellungen der Aufklärung gehörten. "Taten, die man bisher nur für süße liebliche Träume, für fromme theoretische Wünsche guter deutscher Patrioten, für halbe Unmöglichkeiten gehalten."10 Österreich stand damit plötzlich an der Frontlinie der aufgeklärten Nationen. 1781 läßt der Kaiser seine "Grund-Regeln zur Bestimmung einer ordentlichen künftigen Bücherzensur" zirkulieren, die zur Grundlage einer für seine Zeit überraschend großzügigen Literaturpolitik der achtzigerer Jahre werden sollte.

Die Zensur wurde zentralisiert und damit zu einer staatlichen Institution, nachdem sie vorher von der Kirche verwaltet wurde. Als Zensoren werden führende Wiener Aufklärer berufen wie der Schriftsteller Aloys Blumauer oder Gottfried van Swieten. Für wissenschaftliche Werke wird die Zensur aufgehoben, ebenfalls für protestantische Bücher. Die Zahl der verbotenen Bücher sinkt in dem 1783 fertiggestellten Katalog von 5000 auf 900, dazu gehören nun auch traditionelle religiöse Bücher. Die neue Zensurpolitik bedeutet noch keine Pressefreiheit im modernen Sinne, doch signalisierte sie einen deutlichen Bruch mit dem kirchlich dominierten Autoritätssystem unter Maria-Theresia, bei dem jeder Reisende, selbst wenn er "nur von seinen Landgütern in eine Stadt kommt, alle seine Truhen und Bett-Säcke" der Polizei öffnen mußte. Fanden sie ein Buch, das sich auf dem Index befand, wurde es verbrannt, für verdächtige Bücher wurden Gutachten angefordert, sodaß der Reisende oft Wochen auf die Entscheidung der Zensoren warten mußte. ${ }^{11}$

Die neue Zensurpolitik signalisierte jedoch noch mehr, es war ein Bruch mit der herrschenden Barockkultur, die man nicht auf eine Kunst- und Architekturperiode einschränken darf, denn sie war die repräsentative theologische und politische Ausdrucksform der Gegenreformation und sie ${ }^{10}$ Johann Pezzl: Faustin oder das philosophische Jahrhundert, S. 322f.

${ }^{11}$ Resolution Josephs II: "Grund-Regeln zur Bestimmung einer ordentlichen künftigen Bücherzensur". Abgedruckt in: Der Josephinismus. Ausgewählte Quellen zur Geschichte der theresianisch-josephinischen Reformen, hrsg. von Harm Klueting, Darmstadt 1995, S. 215ff. 
vereinte das katholische Europa mit Italien, Frankreich und Spanien. "Die Barockkultur der Gegenreformation war überwiegend unliterarisch und unreflektiert; sie förderte Repräsentation weltlicher und kirchlicher Macht in Musik, Architektur und Theater, tolerierte subliterarische, traditionelle Volkskunst und scholastisch gebundene Wissenschaftspflege, unterdrückte aber jede Form der Literatur und Philosophie, die autonomes kritisches Denken gefördert hätte. Im Modernisierungsprozeß der Donaumonarchie erhält jedoch das geschriebene Wort eine besondere Bedeutung. Regiert wird durch Akten, Berichte und Formulare; die Pflege und Reform der deutschen Sprache wird eine der Grundbedingungen der neuen zentralisierten Administration." 12 Die Literatur wird dabei bewußt für die Zwecke der Reformpolitik Josephs II. - besonders für seinen Kampf gegen die Kirche - instrumentalisiert. Seine Rechnung schien dabei zu Beginn auch voll aufzugehen.

Unmittelbar nach der Veröffentlichung des Zensurdekrets erlebte Wien eine Explosion von Broschüren, Periodika und kleinen Flugschriften aller Art, wie sie innerhalb der deutschsprachigen Literatur des achtzehnten Jahrhunderts einzigartig ist und diese Schriften waren zum größten Teil projosephinisch. ${ }^{13}$ Bereits 1781 berichtet die Allgemeine Deutsche Bibliothek über das neue Phänomen: "Alle Pressen waren beschäftigt, und hundert Schriften kamen, wie Schwämme [Pilze] nach einem fruchtbaren Regen, gleichsam über Nacht hervor; freilich meist Dingerchen, die nicht lange leben [...] Aber, wenn die Pressefreiheit ihren gesegneten Fortgang hat, wenn man nicht, nachdem man den ersten Schritt getan, zurückschreitet, so wird auf die Dämmerung mit der Zeit ein heller Tag folgen, und man kann bessere Schriften, wichtigere Produkte des Geistes und die Umbildung des Geschmacks, die Aufklärung einer ganzen Nation mit Zuversicht erwarten."14

In den Broschüren werden in knapper Form die zentralen Fragen der josephinischen Reformpolitik aufgegriffen: Die Unterordnung der Kirche unter den Staat, das Toleranzedikt und die Aufhebung der Klöster und

${ }^{12}$ Leslie Bodi: Tauwetter in Wien. Zur Prosa der österreichischen Literatur 1781-1795, Frankfurt a.M. 1977, S. 39.

${ }^{13}$ Vgl. dazu: Leslie Bodi: Tauwetter in Wien. Zur Prosa der österreichischen Literatur 1781-1795, Frankfurt a.M. 1977.

${ }^{14}$ Allgemeine Deutsche Bibliothek, hrsg. von F. Nicolai, Berlin 1781, S. $268 \mathrm{f}$. 
Mönchsorden. Mit den Toleranzpatenten wurden den nichtkatholischen Religionsgemeinschaften, wie den Lutheranern, Calvinisten und Orthodoxen und Juden ihre ungehinderte Religionsausübung garantiert, den Juden auch ihre bürgerliche Gleichstellung. Zur Agrarreform Joseph II. gehört die Aufhebung der Leibeigenschaft in Ungarn, die Ablösung der Frondienste durch Geldabgaben und Abschaffung wirtschaftrlicher und rechtlicher Privilegien des Adels. Österreich, einst die Bastion der Gegenreformation nördlich der Alpen, sozial und ökonomisch rückständig, beherrscht von den Jesuiten, die bis 1774 das Erziehungswesen kontrollierten, begann sich in einem Tempo zu wandeln, das die Elite Europas in Begeisterung oder zumindest in Erstaunen versetzte.

\section{3. "Es leben die großen Städte! Sie machen aus Barbaren Menschen ..."15}

Wièn, das einzige wirklich urbane Zentrum des deutschsprachigen Raumes, sollte von den geschilderten Veränderungen am meisten profitieren. Die modernisierenden Reformen Joseph II. brachten das städtische Leben der Hauptstadt buchstäblich zum Tanzen, während sich an der Peripherie des Herrschaftsgebietes in Ungarn und in den österreichischen Niederlanden Gegenkräfte sammelten, die die stürmische Reformperiode bereits 1790 zum Stoppen brachten. Wien wurde zum Schmelztiegel der verschiedenen Nationen, Völker und Kulturen, die die österreichische Monarchie beherbergte, die von der belgischen Kanalküste bis nach Siebenbürgen, von Böhmen bis Mailand und von Freiburg im Breisgau bis Galizien reichte. Das später unregierbare Völkergemisch aus Deutschen, Ruthenen, Italienern; Slowaken, Rumänen, Tschechen, Polen, Ungarn, Slowenen, Kroaten, transsilvanischen Sachsen und Serben bildete in Wien jene bunte Erscheinungsfülle, die jedem Fremden als

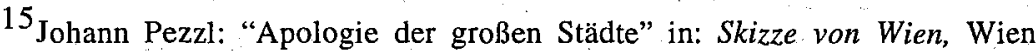
und Leipzig 1786, S. 34f. Pezzl hofft, daß sich die Einwohnerzahl Wiens bald von 265000 auf eine halbe Million erhöhen werde, um im richtigen Verhältnis zu den zwanzig Millionen Menschen der "österreichischen Staaten" zu stehen. Gegen Rousseau verteidigt er die zivilisatorische Wirkung der Urbanisierung. Die Einwohnerzahl wird von heutigen Historikern meist niedriger geschätzt, um 210000 . Paris war nach der Einwohnerzahl doppelt so groß wie Wien und London hatte die vierfache Anzahl von Einwohnern. 
erstes auffiel. Fülle und Buntheit des Straßenbildes wurden noch gesteigert durch die täglichen Feste und Umzüge, die von der Kirche und vom Hof auf den Straßen veranstaltet wurden. Die Enge der Altstadt täuschte dabei noch eine größere Dichte vor.

Nur einen kurzen Blick erlaubte sich der protestantische Aufklärer Georg Forster auf das "häßliche, traurige Bild des Aberglaubens", das sich seit den frühen Morgenstunden vor seinem Fenster abspielte: "Schon seit mehreren Stunden wimmelts gerade vor meinem Fenster vor dem Eingang der Kapuzinerkirche, die heute seit Aufgang der Sonne schon Ablaß für die vergangenen und zukünftigen Sünden verkauft haben. Das arme blinde Volk kniet schon mitten auf der Straße, nur das Gesicht nach der Gegend gerichtet, wo seine Verblender ihm seine Götzen aufstellen." ${ }^{\text {"6 }}$ Nüchtern zählt Nicolai dagegen die vielfältigen Anläße für das Gedränge auf den Straßen auf: "Geistliche und weltliche Gelegenheiten, Leute auf die Straße $\mathrm{zu}$ bringen, sind daselbst ungemein häufig. Prozessionen, öffentliche Segen, feierliche Hochämter und andere geistliche Solennitäten [Feierlichkeiten]; dann Lehnserteilungen und dazu gehörige Aufzüge, Galatage, dahin gehörige Feste, große Gastereien, Besuche und das ganze damit verbundene Schwärmen der modischen Welt, Schauspiele, Tierhetzen, Maskeraden, das Hin- und Hergehen so vieler Handwerker, Tagelöhner, Bedienten, die aus den Vorstädten sich in die Stadt berufswegen begeben müssen, alles dies setzt die Einwohner Wiens mehr in Bewegung, als die Einwohner irgend eines andern Orts."17

Wien, seit 1611 Residenz des Kaisers des Heiligen Römischen Reiches und Hauptstadt aller österreichischen Länder, war Sitz der Zentral- und Mittelbehörden für den von den Habsburgern beherrschten Bereich: der Reichshofrat und die Reichshofkanzlei, die Hofkammer und der Hofkriegsrat, endlich die Regierung der niederösterreichischen Länder. Die leitenden Beamten dieses immer mehr anwachsenden Behördenapparates entstammten dem Adel oder stiegen, wenn es sich um bürgerliche Juristen handelte, sehr rasch in den Adel auf, und zwar oft in den Herrenstand. Neben ihnen steht eine Schicht von Schreibern und Dienern, die mit ihren Familien einen erheblichen Anteil an der Stadtbevölkerung gehabt haben

${ }^{16}$ Georg Forsters Werke, 14. Bd., S. 145. Georg Forster an Therese Heyne, Wien den 1. August 1784.

${ }^{17}$ Friedrich Nicolai: Beschreibung einer Reise durch Deutschland und die Schweiz, 3. Bd., Berlin und Stettin 1784, S. 169. 
müssen. Der reiche Adel mit riesigen Besitzungen in den österreichischen und böhmischen Ländern begann sich seit 1660 in Wien niederzulassen, kaufte ganze Häuserkomplexe auf und errichtete große Adelspaläste. Dazu kamen noch die Sommerpaläste in dem weiten Kranz der Vorstädte, mit ihren Gärten und Parks. Dieser Aufwand war immer auch begleitet von einem Heer von Personal, das ebenfalls zur Repräsentation gehörte.

Die Residenz des Kaisers zog zudem noch Gesandte und Abgeordnete von allen deutschen Fürsten nach Wien. Der Reichshofrat, eines "der höchsten Reichsgerichte", zwang die an den Prozessen Beteiligten, in die Stadt zu kommen, und da die Prozesse oft lange dauerten, mußten sich die Bittsteller auf einen langen Aufenthalt in der Stadt einrichten. ${ }^{18}$ Allen diesen Schichten gegenüber besaß das eigentliche Bürgertum Wiens - wie Otto Brunner feststellt - "lange kein wirkliches Gesicht." Wien, "das als Herrscherresidenz, als "Kaiserstadt" verstanden wurde," wies "einen starken aristokratischen Einschlag in seiner Sozialstruktur auf."19 Von einer einheimischen großbürgerlichen Schicht kann in Wien erst von der Mitte des achtzehnten Jahrhunderts gesprochen werden. "Aber diese bürgerliche Oberschicht fügt sich doch im wesentlichen den geadelten Offiziers- und Beamtenschichten an, die in Mitteleuropa, anders als in Westeuropa, lange ein höheres soziales Prestige als das Bürgertum behauptet haben." 20

Das bunte Treiben, das man in Wien auf den Straßen sah und das den Besucher durch die fremden Trachten beeindruckte, bestand zum großen Teil aus der Aristokratie des Reiches oder der Monarchie, sie bildete durch ihren Lebensstil, der durch eine gemeinsame Gesellschaftsethik geprägt war, die Klammer, die diese multikulturelle Welt zusammenhielt. "Es wird keine Stadt sein, außer allenfalls Amsterdam," schreibt Nicolai, "wo man so vielerlei Nationen zusammensieht, als in Wien; und gewiß ist keine, wo man so vielerlei Sprachen reden hört als dort. Eine Menge Deutscher aus allen deutschen Ländern, besonders aus den südlichen, strömt nach Wien. Es ist unglaublich, wie viel fremde Menschen vom

${ }^{18}$ Ebd., S. 170.

${ }^{19}$ Otto Brunner: "Hamburg und Wien. Versuch eines sozialgeschichtlichen Vergleichs" in: Otto Brunner: Neue Wege der Verfassungs- und Sozialgeschichte, Göttingen 1968, S. 334.

20 Otto Brunner: "Zwei Studien zum Verhältnis von Bürgertum und Adel", Ebd., S. 280. 
gemeinen Volke nur aus dem Reiche durch schwäbische und baierische Schiffe wöchentlich dahin gebracht werden. [...] Wien gefällt den Fremden; und an Nahrung und gutherzigen Leuten fehlts auch nicht: also lassen sie sich daselbst nieder und leben so gut sie können. Außer den Deutschen sieht man Italiäner, Schweizer, Elsasser, Böhmen, Mähren, Polen, Russen. Besonders bemerkt man viele Ungarn und Siebenbürger; welche sich zwar seit vierzig Jahren sehr dem Hofe genähert haben, dennoch aber noch immer Nationalgeist und Liebe zum Vaterlande in hohem Grade besitzen, wovon eines der geringsten Zeichen ist, daß sie ihre ohnedies so schöne Nationaltracht beständig beibehalten. Ebenso sieht man viele Nachbarn Ungarns in ihrer Nationalkleidung, als Illyrier, Raizen [Serben], Wallachen (oder Romaner), Bulgaren aus der Moldau, Griechen; besonders auch Türken, deren sich beständig einige der Handlungen [des Handels] wegen in Wien aufhalten; auch wohl Armenier, Maroniten u. a. Unter den Niederländern, die sich hier aufhalten, sind verschiedene der berühmtesten gelehrten Namen [...] Franzosen finden sich in ziemlicher Anzahl in Wien, wo finden sich die nicht? Auch von ihnen sind viele Meßpriester, Informatoren [Hauslehrer], und französische Mamsellen, deßgleichen Kammerdiener, Friseurs, Köche, Glücksritter und Schriftsteller. Die Juden, welche unter dem jetzigen Kaiser die Freiheit erhalten haben, Handwerke und freie Künste zu treiben, wodurch diese Nation gewiß dem Staate nützlich werden kann, sind bis jetzt noch nicht in großer Anzahl."21

\section{Räume der Gleichheit: Freimaurerlogen und Salons}

Bereits am ersten Tag in Wien wird Georg Forster mit der neuen demokratisierten Form aristokratischer Festkultur unter Joseph II. vertraut gemacht: Feuerwerk und Ballonaufstieg im Prater, auch der Kaiser ist anwesend. Neben dem Augarten hatte Joseph II. 1766 auch den Prater dem allgemeinen Publikum zugänglich gemacht, der vordem nur dem Hof offenstand. Der Prater entwickelte sich schnell zu einem beliebten Ausflugsziel, Gasthäuser, Kaffeehäuser, Tanzsäle, Kegelbahnen sorgten neben einem regelmäßig veranstalteten Feuerwerk für zusätzliche Unterhaltung. "Die Feuerwerkstage sind die schönsten Tage des Praters. Der Eintritt

${ }^{21}$ Nicolai: Beschreibung einer Reise, Bd. 3, S. $170 \mathrm{ff}$. 
kostet zwanzig Kreuzer; dies macht, daß bei diesem Schauspiel der geringe Pöbel wegbleibt, und dann nur das bessere Publikum erscheint. Gegen fünf Uhr Abends fängt der Zug dahin an. [...] Man macht erst eine kleine Spatzierfahrt im Walde, oder bestellt sich nach Wienersitte eine Jausen unter den Bäumen."22

Forster fuhr mit dem Verleger, Buchhändler und aktiven Freimaurer Rudolph Gräffer, der seinen Wiener Aufenthalt vorbereitet hatte, zum Prater. Durch ihn wurde Forster in die Kreise der Wiener Freimaurer und in die wichtigsten Salons eingeführt, was ihm die Türen $\mathrm{zu}$ allen wichtigen Persönlichkeiten der Stadt - bis hin zum Kaiser - öffnen sollte. Er lernte dabei in wenigen Tagen die verschiedenen Institutionen der eben entstehenden Öffentlichkeit für sich zu nutzen. Die zentrale Erfahrung, die Forster an allen diesen Orten machte, war, daß im Jahre 1784 "der Traum von einer bürgerlichen Republik, in der Adlige, Schriftsteller und Künstler als Gleiche miteinander verkehren, [...] für die österreichische Intelligenz [...] für einen Augenblick Wirklichkeit zu werden schien."23 In den Freimaurerlogen und in den Salons lernte er Formen "gemeinschaftlicher Gleichheit" 24 kennen, die ihm in dieser Offenheit bisher nicht begegnet waren. In der ersten Hälfte der achtziger Jahre entstanden in Wien mehr und mehr Orte, an denen ein sozialer Verkehr über Standesgrenzen hinweg, eine Annäherung des Lebensstils und der Weltanschauung möglich wurde, wo ständische Privilegien abgebaut wurden und sich die Grenzen zwischen den Ständen zu verwischen begannen.

Was die Freimaurerei betriff, so kam Forster als gebranntes Kind nach Wien. In Kassel war er kurz vorher in einer Rosenkreuzerloge in den finstersten Mystizismus geraten und zudem noch schmählich betrogen worden. ${ }^{25}$ Nur zögernd ließ er sich erneut auf die Freimaurerei ein und stellt den "Brüdern" immer wieder bohrende Fragen über das Verhältnis von Freimaurerei und Aufklärung. In seinem Tagebuch heißt es dann: "Dann hinüber zu Born und mit ihm gesprochen bis nach halb 10 von

${ }^{22}$ Johann Pezzl: Skizze von Wien. Wien und Leipzig 1786ff. Zitiert nach Joachim Schondorf (Hrsg.): Aufklärung auf wienerisch, Wien, Hamburg 1980, S. 68.

${ }^{23}$ Roger Bauer: Die Welt als Reich Gottes, Wien 1974, S. 49.

${ }^{24}$ Vgl. Richard Münch: Die Kultur der Moderne, 2 Bd., Frankfurt a.M. 1986.

${ }^{25}$ Vgl. dazu Gerhard Steiner: Freimaurer und Rosenkreuzer - Georg Forsters Weg durch Geheimbünde, Berlin 1985. 
Maurerei" - "um 9 zu Baron Gemmingen. Über Maurerei."26 Am Ende wurde er jedoch davon überzeugt, daß die von ihm so verhaßte Schwärmerei und Alchimie in den bedeutendsten Logen nur eine marginale Rolle spielten. Von seiner ersten Begegnung mit Logenbrüdern notiert er zusammenfaßend: "Kurz eine Gesellschaft von 17 munteren, lebhaften, freundschaftlichen und untereinander durch Liebe und Freundschaft verbundenen Leuten, die den Samen der Aufklärung ausstreuen, Toleranz zu predigen, den Vorurteilen Stirne zu bieten, und über alles freimütig zu sprechen und zu denken gewohnt sind."27

Das egalitäre Element der freimaurerischen Binnenorganisation wird in einer Freimaurerschrift von 1744 folgendermaßen charakterisiert: "Sobald wir versammelt sind, werden wir alle Brüder [...] Der Fürst, der Untertan, der Edelmann und Bürger, der Reiche und der Arme, ist einer so gut als der andere, nichts unterscheidet sie von einander und nichts trennet sie. Die Tugend macht sie alle einander gleich."28

In dem geschlossenen Kreis der bedeutendsten Loge "Zur wahren Einheit", der Forster hier beiwohnt, scheint das beschriebene Ideal Wirklichkeit geworden zu sein, es herrscht eine von familiärer Wärme durchströmte Brüderlichkeitsethik, die ihn sofort anzieht. Ignaz von Born, der führende Kopf der österreichischen Freimaurerei und vielleicht das Vorbild des Sarastro in Mozarts Zauberflöte, konnte bei der Sitzung nicht anwesend sein, von ihm heißt es im Tagebuch: "Es wurde hier, da es Ignatii Tag war, des lieben Borns Namenstag gefeiert. Ich mußte unzählige mal hören, wie lieb er mich hätte, wie sehnlich und schmerzlich er auf meine Ankunft geharrt habe [...] Die Liebe, die jeder für ihn hat, ist unbeschreiblich. Ein Vater unter lauter liebenden und geliebten Kindern ist er." 29

Born, selbst ausgebildeter Mineraloge, Hofrat bei der Hofkammer in Münz- und Bergwerkssachen, und besonders interessiert an einer durch

${ }^{26}$ Georg Forsters Werke, 12. Bd., Tagebücher, S. 113 und 115.

${ }^{27}$ Ebd., S. 105.

${ }^{28}$ Der sich selbst vertheidigende Freymäurer oder Sammlung unterschiedlicher, wohl verfaßter Schriften, welche einige Mitglieder dieses Ordens selbst $z u$ dessen Vertheidigung herausgegeben, nebst einer vorläufigen historischen Nachricht von dieser vortrefflichen Gesellschaft, Frankfurt und Leipzig 1744, S. 205.

${ }^{29}$ Georg Forsters Werke, 12. Bd., Tagebücher, s. 105. 
Technik und Wissenschaft verbesserten Industrie, ${ }^{30}$ verstand es, die Loge "Zur wahren Einheit" in kürzester Zeit zum Versammlungsort der bedeutendsten Wiener Aufklärer zu machen. "His purpose at "Zur wahren Einheit" was to fulfil a long-held dream to create in Vienna an academy of intellectual and scientific enquiry to match the famous Royal Society in London, which had itself, through Desaguliers, been closely involved in early English freemasonry and Newtonian Deism."31

Durch seinen charismatischen Führungsstil gelang es Born, die wichtigsten hohen Staatsbeamten, Professoren und Literaten, die die josephinische Reform unterstützten, um sich zu sammeln. Das Charakteristische der Wiener Aufklärung ist jedoch, daß es keinen namhaften Schriftsteller, Staatsbeamten, Aristokraten oder Bürgerlichen gab, der die Ziele der Aufklärung teilte und der nicht der einen oder der anderen Loge angehörte. Dabei wurden in den Logen der Monarchie nicht nur Standesschranken virtuell aufgehoben, sondern auch nationale und konfessionelle Zugehörigkeiten: Deutsche wie Ungarn, Tschechen, Polen und Italiener waren in den Logen organisiert. In einem Brief berichtet Forster ausführlicher über seine Erfahrungen mit der Freimaurerei: "Die Maurerei geht in vollem Schwunge. Alles ist Macon. Alle kaiserlichen Logen sind vereint unter einem gemeinschaftlichen Haupte [...] Die Loge zur wahren Eintracht ist diejenige, welche am allermeisten zur Aufklärung wirkt. Sie gibt ein Journal für Freimaurer heraus, worin über Glauben, über den Eid, über die Schwärmerei, über die Ceremonien, kurz über alles freier gesprochen wird, als man bei uns, d.h. in Niedersachsen herum, tun würde. Die besten Köpfe Wiens unter den Gelehrten, und die besten Dichter sind Mitglieder drinnen. Man spottet darin über alles was Heimlichkeit bei der Sache ist, und hat die ganze Sache zu einer Gesellschaft wissenschaftlicher, Aufklärung liebender, von Vorurteil freier Männer umgeschaffen, Born ist Meister vom Stuhl darin.,"32

Geselliger Höhepunkt im Kreise der Freimaurer war Forsters Aufnahme in die Loge "Zur wahren Einheit" als "Meister" und die sich daran anschließende Tafelloge zu seinen Ehren. "Ich bin am Sonntag entsetzlich

${ }^{30}$ Österreich, einer der größten Eisenproduzenten der Zeit, hatte das größte Eisenwerk der Welt.

${ }^{31}$ Nicholas Till: Mozart and the Enlightenment, London, Boston 1992, S. 121.

${ }^{32}$ Georg Forsters Werke, 14. Bd., Briefe, S. 162f. 
fétiert [gefeiert] worden." berichtet er seinem Freund Sömmering, "Born hielt eine Tafelloge von 84 Couverts [Gästen], wo der NationalGroßmeister Graf Dietrichstein, der Baron von Gebler (hier einer der angesehensten Männer), Gemmingen und eine Menge andere Besuchende zugegen waren." 33

Die Freimaurerei hatte zwei Pole: Die Absonderung im esoterischen Kreis und die Pflege eines internationalen Kommunikationsnetzes. Die festliche Geselligkeit mit dem Weltumsegler Forster war Teil dieses internationalen Programms. Die Behauptung ein "jedes Mitglied des Ordens (habe) einen Zutritt bei allen Logen in der Welt", ${ }^{34}$ hatte einen konkreten kosmopolitischen Kern und konnte mehr wert sein als eine Kreditkarte heute. Der Kreis um Born pflegte intensive Kontakte zu norddeutschen Aufklärern, besonders zu Martin Wieland in Weimar. Eine Schlüsselrolle bei der Verbindung Wiens mit der norddeutschen Aufklärung spielte Karl Leonhard Reinhold, der ab 1786 mit seinen Briefen über die Kantische Philosophie entscheidend zur Breitenwirksamkeit Kants beitrug. Reinhold, in Wien 1758 geboren, Exjesuit, war seit 1782 Mitglied der Loge "Zur wahren Einheit", bis er nach Weimar ging und mit Wieland den Teutschen Merkur herausgab, nach 1788 lehrte er dann das "Evangelium der reinen Vernunft" in Jena. Seine Verbindung mit Wien riß jedoch nicht ab, und Reinhold publizierte weiter in Wien.

Deutlich wird bei der Lektüre von Forsters Tagebuch, daß die Gesellschaft, die den Weltumsegler in ihren Kreisen feierte, zwischen zwei Brennpunkten oszillierte: der Freimaurerloge und dem Salon. Beide Institutionen spielen für die Wiener Aufklärung eine zentrale Rolle, dagegen trat die Universität, die Wien ebenfalls beherbergte, in den Hintergrund. Beiden Institutionen ist gemeinsam, daß sie für einen exklusiven Kreis zur Begegnungsstätte von bislang voneinander getrennten Ständen und Schichten werden.

Joseph II. hatte im Rahmen seiner rigorosen Sparpolitik die Aufgaben des Hofes auf ein Minimum reduziert, Feste am Hof gab es nur, wenn es unvermeidlich war, etwa beim Besuch wichtiger Staatsgäste. Durch den Funktionsverlust des Hofes bekamen die adligen und bürgerlichen Salons einen bedeutenden Auftrieb, da sich das gesellschaftliche Leben nun in den

${ }^{33}$ Ebd., S. 166.

${ }^{34}$ Der sich selbst verteidigende Freymäurer, S. 117. 
Palästen des Adels oder den Häusern der reichen Bürger abspielte. Wichtig war hier jedoch für unseren Problemzusammenhang, daß die Gesetze des Hofes andere waren als die des Salons. Der Hof stand unter dem Zwang des Zeremoniells, das jede Handlung bis hin zur Kleidung regelte. Aufgabe des Zeremoniells war es, den gesellschaftlichen Rang jeder darin einbezogenen Person sinnfällig zu machen. Dieses Hofzeremoniell wirkte auch über den engen Bereich des Hofes hinaus verhaltensprägend: In dem Maße, wie der höfische Lebensstil allgemein als vorbildlich akzeptiert wurde, begann auch das städtische Bürgertum, seine Formen zu kopieren und z.B. den geringsten Rangunterschied penibel hervorzuheben, was zur sozialen Fragmentierung der städtischen Gesellschaft beitrug.

Tendenziell egalitär ist dagegen der Umgang im Salon für die Dauer des Zusammenseins: "Kaum merkt mans, daß man unter Leuten von Stande ist," schreibt Forster an seine Verlobte, "und jeden Augenblick möchte man es vergessen, und sie auf den vertrauten Fuß der gleichgeborenen Freunde behandeln." ${ }^{35}$ Hier gab es kein steifes Zeremoniell, keine Rangfragen, keine geregelten und einzuhaltenden Besuchszeiten, aber eine zu jeder Stunde geübte Gastlichkeit. Der Salon war das Königreich einer Frau, sie bestimmte durch ihre Einladungen die Zusammensetzung der Gesellschaft, sie gab als Dirigentin unauffällig das Zeichen für den Einsatz der Stimmen und der Themen; sie sorgte durch ihre Aufmerksamkeit für das Gefühl selbstverständlicher Zugehörigkeit bei einem neuen Gast. Für Forster, wie vor ihm schon für Mozart wird der Salon der Gräfin Maria Wilhelmine von Thun zum Sprungbrett in den innersten Kreis der herrschende Gesellschaft. "Fast alle Abend zwischen neun und zehn Uhr kommen diese Leute bei der Gräfin Thun zusammen, da wird allerlei witziges Gespräch geführt, es wird Clavier gespielt, deutsch oder italienisch gesungen, auch wohl, wenn die Begeisterung die Leute überfällt, getanzt. Stellen Sie sich ihren unbeholfenen Forster vor, wie er [...] trotz aller Protestation am Arme gepackt und von einer Tänzerin zur andern geschoben wurde."36

Der Wiener Salon setzte unter der Führung des Adels die große Tradition der französischen Gesprächs- und Erzählkultur fort, er kultivierte Esprit, die sprachliche Eleganz, Expressivität in Musik und Tanz, Kreativität und

${ }^{35}$ Georg Forsters Werke, 14. Bd., Briefe, S. 180.

${ }^{36}$ Ebd. S. 181. 
Scharfsinnigkeit. Neben der Musik wurden Sprachkenntnisse hoch veranschlagt: "Französisch und Italienisch kann Jedermann; und zum Erstaunen viel können Englisch." 37 Alles dies sind Fähigkeiten, die in der von der Universität geprägten norddeutschen Denkkultur eine geringere Rolle spielten. Doch die Salonkultur in Wien, die jeden Abend neu entstand und wieder verging, hat keine theoretischen oder literarischen Werke hinterlassen, die heute noch einen Einfluß ausüben. Nur Experten kennen heute die Namen der Schriftsteller und Philosophen, die die Wiener Aufklärung ausmachten.

\section{5. "Was in unsern Zeiten nicht erlaubt ist, gesagt zu werden, wird gesungen."}

Die Fülle, der so flüchtigen und so kurzen Blüte der Wiener Aufklärung ist jedoch in dem Werk eines Mannes eingefangen, das die Tradition der europäischen Musikkultur mit der neuen Philosophie auf eine für die Zeitgenossen schwer verständliche Weise verband. Sein Name taucht in den Tagebüchern und Briefen Forsters kein einziges Mal auf, auch wenn dieser Mann in denselben Salons verkehrte, dieselben Freunde hatte und ebenfalls - wenn auch erst drei Monate später - in denselben Freimauererlogen verkehrte. Es ist Wolfgang Amadeus Mozart.

Die Oper, der Ort festlicher Öffentlichkeit eines priviligierten Publikums, wurde durch Mozart zum Sprachrohr für die neuen Ideen. Die politische Aggressivität seiner 1785 entstandenen Oper Le nozze di Figaro ist getragen von dem Optimismus der frühen achtziger Jahre in Wien. Der Bericht der Wiener Realzeitung über die ersten Aufführungen im kaiserlich königlichen National- und Hoftheater bringt die singuläre Situation Wiens auf den Punkt: "Was in unseren Zeiten nicht erlaubt ist, gesagt zu werden, wird gesungen. [...] Dieses Stück, das man in Paris verboten, und hier als Komödie sowohl in einer schlechten als in einer guten Übersetzung aufzuführen nicht erlaubt hat, waren wir endlich so glücklich als Oper vorgestellt zu sehen. Man sieht, daß wir besser daran sind als die Franzosen." 38 Mozart hatte zusammen mit dem Hofpoeten Lorenzo Da Ponte die satirische Gesellschaftskomödie La folle journée ou le mariage

${ }^{37}$ Ebd. S. 173.

${ }^{38}$ Wiener Realzeitung vom 11. Juli 1786. Zitiert nach Volkmar Braunbehrens: Mozart in Wien, München 1986, S. 301. 
de Figaro [Der tolle Tag oder Figaros Hochzeit] von Beaumarchais in ein Musik-Theater umgeformt, das wie nie zuvor zeitgenössische soziale Alltagskonflikte realistisch und unverblümt auf die Bühne brachte. Präzis plaziert Mozart seine Oper in die tägliche Konfliktzone einer aristokratischen Gesellschaft, deren Existenz ohne die Dienste des Personals zusammenbrechen würde. Er nimmt Partei für die Schicht, die dafür angestellt wurde, "gewisse Gefäße, die zu heimlichen Verrichtungen gebraucht werden, ihres Inhalts zu entledigen, Wände abzustauben, Zimmerböden zu säubern, Tische und Kästen zu wixen etc., etc. mit einem Wort Dienstboten zu sein." ${ }^{39}$ Es geht in der Oper um die Selbstbehauptung einer Gruppe, denen das Recht auf autonome Gestaltung ihre Lebens systematisch vorenthalten wurde. An ein Heiraten ohne die Einwilligung des Herren war unter den Bedingungen des feudalen Rechts nicht zu denken. ${ }^{40}$

Die Oper zeigt das Dienerpaar Figaro und Susanna im Zustand des Glücks, erfüllt von Vorfreude bei der sehr nüchtern-handwerklichen Vorbereitung der Hochzeit. Susanna probiert ihr selbstgemachtes Hütchen an, Figaro vermißt den Raum für das Bett. Die erotisch-heitere Szene wird abrupt zerstört durch die Aufdeckung der Verführungspläne des Grafen durch Susanna. Gewalt (die Musik stattet den Grafen mit barockem, herrischen Gestus aus) und List gefährden das paradiesische Glück des Paares. Gewährte "grazie" (Gunst) und "cura" (Fürsorge) durch den Herrn verwandeln sich nach dieser Entdeckung mit einem Schlag zu einer Bedrohung.

Das komplizierte Intrigenspiel beginnt mit der wilden Auflehnung Figaros gegen den Grafen, die sich nur widerwillig in das kalte Kalkül der Intrige pressen läßt, die allein Erfolg verspricht. Die Rebellion artikuliert sich zuerst in einem imaginierten Zweikampf, bei dem Figaro den Rhythmus bestimmt: "Wenn sie tanzen wollen, liebes Gräflein, werde ich ihnen das Gitarrchen schlagen." Die Musik gibt hier ein eindeutiges politisches Signal, der Vertreter des niedrigen Standes eignet sich selbstherrlich das höfische Menuett an, um die Puppen nach seinem Takt

${ }^{39}$ Johann Rautenstrauch: Über die Stubenmädchen in Wien, Wien 1781. Zitiert nach Joachim Schondorf (Hrsg.): Aufklärung auf wienerisch, Wien, Hamburg 1980, S. 239 f.

${ }^{40}$ Vgl. Nicholas Till: Mozart and the Enlightenment, S. $155 f$. 
tanzen zu lassen. Doch der erste Anlauf unter der Regie Figaros scheitert und nun übernehmen die Frauen, die Gräfin und Susanna, das Heft. In der Turbulanz der Ereignisse wachsen - über die Standesgrenzen hinaus - die Gräfin und ihre Kammerzofe zu einer verschworenen Gemeinschaft zusammen, während der Graf in eine immer größere Isolation gerät, bis er am Ende vor allen Beteiligten überführt wird und unter dem Druck dieser Öffentlichkeit um Verzeihung bitten muß. Der Graf muß lernen, daß er gegenüber der Integrität seines Dienerpaares, das die neuen bürgerlichen Werte vertritt, keine Macht hat. Gegenüber der von Verstellung und Intrige beherrschten aristokratischen Gesellschaft erweist sich das junge Paar, das die Zukunft repräsentiert, als unfähig die Maske aufrechtzuerhalten: Vorgetäuschte Aktionen verwandeln sich unbewußt in aufrechte Gefühle. Feudales Recht, Geld und selbst Intrigen erweisen sich dagegen als stumpfe Waffen.

Die Oper Le nozze di Figaro verbindet genau beobachteten sozialen Realismus, in der der Gesellschaft des ancien régime der Spiegel vorgehalten wird, mit der Utopie von Gleichheit. Das utopische Moment zielt nicht vorrangig auf die Beseitigung von Klassenunterschieden, sondern stellt die kategorische Forderung in den Vordergrund, jedem Menschen das Recht auf die Verwirklichung seiner Gefühle und Sehnsüchte einzuräumen. "In 'Le nozze di Figaro' herrscht historisch und gattungsgeschichtlich gesehen ein einziges, erstes und, betrachtet man die folgenden Opern Mozarts, letztes Mal absolute Gleichheit und Freiheit, derer sich die Frauen weit souveräner und intuitiver bedienen als die Männer."41

Diener und Stubenmädchen waren von den Inseln der Gleichheit, die Salon und Freimaurerloge bildeten, ausgeschlossen. In der sozialen Utopie, die Mozart in Le nozze di Figaro gestaltet, treten sie in den Mittelpunkt. Aus dem Salon übernimmt Mozart jedoch in seine Oper nicht nur die Freude an Witz und Schlagfertigkeit, sondern auch die Erotik, die Forster im Salon der Gräfin Thun überwältigen sollte. Es ist eine Erotik, die offen und allgegenwärtig, bereichert wurde durch die Intensität einer neuen Innerlichkeit.

"Einen tollen Tag und eine tolle Nacht konnte Mozart nur ein einziges Mal schaffen. Nach "Le nozze di Figaro" wird es im Mozart-Theater

${ }^{41}$ Wolfgang Willaschek: Mozart-Theater: Vom Idomeneo bis zu Zauberflöte, Stuttgart 1996, S. 126. 
niemals wieder hell.." ${ }^{22}$ Das Fest, zu dem die Schloßbewohner, der Graf und die Gräfin, der Kammerdiener Figaro und die Kammerzofe Susanna am Ende der Oper aufbrechen, findet in der Oper nicht mehr statt. Es ist als ein Versprechen bis heute noch nicht eingelöst worden: "Geliebte, Freunde, zum Tanz, zum Spiel! / Zündet das Feuerwerk! / Und bei den Klängen eines fröhlichen Marsches eilen wir alle zum Fest."

\section{6. "Wen solche Lehren nicht erfreun, /verdienet nicht, ein Mensch zu sein." 43}

Im Frühjahr 1790 befand sich Georg Forster wieder auf Reisen, diesmal begleitet von dem einundzwanzigjährigen Alexander von Humboldt. Seine Reise, die über Düsseldorf, Aachen in die Österreichischen Niederlande führen sollte, um von dort nach Frankreich und England zu gehen, stand politisch unter dem Zeichen der Französischen Revolution auf der einen Seite und dem Scheitern der Reformpolitik Kaiser Josephs auf der anderen. Forsters Aufenthalt in Brüssel, dem Zentrum des Widerstandes gegen die Reformen, wird für ihn zum Anlaß, über die Ursachen dieses Scheiterns nachzudenken.

Das bunte Völkergemisch im Zentrum Wiens, das die multilinguale und multikulturelle Welt der Habsburger Monarchie widerspiegelte, hatte sich unter dem Diktat der Reformen zu einem Sprengsatz entwickelt, der am Ende der achtziger Jahre die Monarchie zu zerstören drohte. Der Umbau der ganzen österreichischen Monarchie in einen zentralisierten Staat mit einem modernen Herrschaftsapparat rief zuerst an der Peripherie - in Ungarn und in den österreichischen Niederlanden - erbitterten Widerstand hervor. Bei der Vereinheitlichung der Verwaltung wurden die einzelnen Länder mit ihren gewachsenen Traditionen zu bloßen Verwaltungseinheiten herabgedrückt. Die Entmachtung der Stände und die Einführung des Deutschen als Amtssprache in Ungarn dienten dem Ziel einer effektiven Verwaltung. Der Modernisierungsprozeß erstreckte sich auch auf das Gebiet der Gesetzgebung und des Rechtssystems, auch hier ging es um eine Vereinheitlichung. Der aufgeklärte Absolutismus, den Joseph II. vertrat, erwies sich als unfähig, partikularen Ansprüchen gegenüber ${ }^{42}$ Ebd., S. 184.

${ }^{43}$ Sarastro in W. Mozart/E. Schikaneder: Die Zauberflöte. 
Kompromisse einzugehen. Auf den wachsenden Widerstand antwortete er mit dem Aufbau eines Polizei- und Überwachungssystems. 1785 wurden die Freimaurer strengen Regelungen unterworfen und unter Polizeiaufsicht gestellt, "weil jede Versammlung von verschiedenen Ständen der Menschen sich selbst nicht überlassen bleiben kann, sondern unter bekannter Leitung und Aufsicht geprüfter Männer stehen muß."44 Die Überbrückung von Standesschranken wurde zum subversiven Akt. Die Orte der sich gerade konstituierenden Öffentlichkeit, Kaffeehäuser, Salons und die öffentlichen Gärten waren von Spitzeln durchsetzt. Gleichheit stellte sich allein über den Verlust von Freiheitsrechten (der altständischen Gesellschaft) her, Gemeinde-, Städte- und Provinzialversammlungen wird das Recht auf Selbstverwaltung entzogen, um alle Untertanen vor dem absoluten Herrscher einander anzugleichen.

Gleichzeitig mit der Französischen Revolution kommt es im Sommer 1789 in Brabant zur bewaffneten Erhebung, nachdem zuvor die Reformen Josephs als verfassungswidrig erklärt wurden. Im Oktober 1789 bereits wird der Kaiser für abgesetzt erklärt und im Januar 1790 - kurz vor seinem Tod - kommt es zur Vereinigung der zehn Provinzen (Brabant, Flandern, Luxemburg u.a.) unter dem Namen "Etats Belgiques Unis".

Das Scheitern der Reformpolitik stellte die Aufklärung vor gänzlich neue Fragen, da für sie die Nation, die Bewahrung kultureller Eigenständigkeit keinen Wert für sich darstellt. "Wer Josephs Recht, in den Niederlanden nach seiner Erkenntnis des Besseren zu herrschen, in Zweifel zieht, und seine Reform gewalttätig nennt, der darf ihm wenigstens nicht das ursupierte, im Stumpfsinn und im Aberglauben des Volkes geschöpfte Recht der Stände entgegensetzen." ${ }^{45}$ Was Forster Joseph vorwirft, ist die Verletzung der Klugheitsregel, der die Kräfte des Widerstandes unterschätzte und die Reformen zu rasch vorantrieb, doch gelingt es Forster, in seinen Überlegungen noch einen Schritt weiterzugehen: "Die Lieblingsidee des Kaisers, eine völlige Gleichförmigkeit des Administrationswesens und der Gesetzgebung in allen seinen Staaten einzuführen, ist ebenfalls nicht frei von Tadel geblieben. Es scheint in der Tat natürlicher,

${ }^{44}$ Freimaurerpatent Josephs II. (Dezember 1785). Zitiert nach Leslie Bodi: Tauwetter in Wien, S. 230.

${ }^{45}$ Georg Forster: "Ansichten vom Niederrhein, von Brabant, Flandern, Holland, England und Frankreich im April, Mai und Junius 1790" in: Georg Forsters Werke, 9. Bd., Berlin 1958, S. 174. 
die Formen nach dem verschiedenen Genie der Völker abzuändern, als alle Völker in Eine Form zu zwängen. In Italien, Deutschland, Böhmen, Ungarn und Belgien sind die Menschen viel zu weit von einander verschieden in physischen und moralischen Anlagen, in Sitten und Gewohnheiten, um gleichen Handlungen denselben Wert beizumessen. Die Verschiedenheit des Bodens, der Lage, des Himmelstrichs bestimmt diese Mannigfaltigkeit im Menschengeschlecht, wie in der ganzen organischen Schöpfung [...] Sie durch irgend einen Mechanismus einschränken wollen, scheint beinah eine Versündigung an der Natur."46 In Forsters pragmatischem Vorschlag, der weder den historischen Voraussetzungen noch den theoretischen Anforderungen gerecht wird, deutet sich bereits mit der Entgegensetzung von organischer Natur und mechanischem Staat eine romantische Kritik am achtzehnten Jahrhundert an.

Die Feste der Aufklärung in Wien gehören der Vergangenheit an, doch die Probleme, die Österreich durch die Modernisierung seines multilingualen und multikulturellen Herrschaftsbereiches schuf, sind die unseren geblieben. Ihre theoretische und praktische Lösung wird $\mathrm{zu}$ den zentralen Aufgaben der Zukunft gehören.

${ }^{46}$ Ebd., S. 174f. 